\title{
Melting properties from ab initio free energy calculations: Iron at the Earth's inner-core boundary
}

\author{
Tao Sun, ${ }^{1, *}$ John P. Brodholt, ${ }^{2,3}$ Yunguo $\mathrm{Li}^{2},{ }^{2}$ and Lidunka Vočadlo ${ }^{2}$ \\ ${ }^{1}$ Key Laboratory of Computational Geodynamics, College of Earth and Planetary Sciences, \\ University of Chinese Academy of Sciences, Beijing 100049, China \\ ${ }^{2}$ Department of Earth Sciences, University College London, Gower Street, London WC1E 6BT, United Kingdom \\ ${ }^{3}$ Centre for Earth Evolution and Dynamics (CEED), University of Oslo, 0316 Oslo, Norway
}

(Received 26 August 2018; published 3 December 2018)

\begin{abstract}
We present a general scheme to accurately determine melting properties of materials from $a b$ initio free energies. This scheme does not require prior fitting of system-specific interatomic potentials and is straightforward to implement. For the solid phase, ionic entropies are determined from the phonon quasiparticle spectra (PQS), which fully account for lattice anharmonicity in the thermodynamic limit. The resulting free energies are nearly identical (within $10 \mathrm{meV} /$ atom) to those from the computationally more demanding thermodynamic integration (TI) approach. For the liquid phase, PQS are not directly applicable and free energies are determined via TI using the Weeks-Chandler-Andersen (WCA) gas as the reference system. The WCA is a simple, short-range, purely repulsive potential with established equation of states. As such, it is an ideal reference for $a b$ initio TI of liquids. We apply this scheme to determine melting properties of hexagonal close-packed (hcp) iron at the Earth's inner core boundary $(P=330 \mathrm{GPa})$, a subject of great significance in Earth sciences. The important influences of system size and pseudopotentials are carefully analyzed. The results (melting temperature equals $6170 \pm 200 \mathrm{~K}$, latent heat $56 \pm 2 \mathrm{~kJ} / \mathrm{mol}$, Clapeyron slope $8.1 \pm 0.2 \mathrm{~K} / \mathrm{GPa}$ ) are consistent with experiments as well as previous calculations.
\end{abstract}

DOI: 10.1103/PhysRevB.98.224301

\section{INTRODUCTION}

The melting properties of materials are important in many fields, including metallurgy [1], nuclear technology [2], chemical engineering [3], etc. In the context of Earth sciences, melting properties of minerals form the basis for understanding the Earth's thermal structure, dynamics, and evolution [4,5]. For instance, the melting temperature $\left(T_{m}\right)$ of iron at the Earth's inner-core boundary (ICB) is an important anchor for estimating the temperature at the center of the Earth $[6,7]$. Melting properties of silicates dictate magma formation [8], igneous differentiation [9], and other key geological processes [10]. In spite of this importance, melting properties of minerals at conditions of the Earth's deep interior have not been well constrained [11]. Experimental measurements at high pressures $(P)$ and temperatures $(T)$ are challenging and the results are hampered by substantial uncertainties. Indeed, $T_{m}$ of iron at ICB conditions $(P=330 \mathrm{GPa}$ and $\sim 6000 \mathrm{~K})$ inferred from various experiments ranges from 4850 to $7600 \mathrm{~K}$, a difference of nearly $3000 \mathrm{~K}$ [11]. The large discrepancy is partly due to competing phenomenological criteria adopted to identify the onset of melting [7], and a consensus is yet to be reached on which criterion is more appropriate [11]. As well as experiments, melting properties can also be determined by $a b$ initio free energy calculations [12-14]. Such calculations are known to exhibit good accuracy in spite of extreme $P-T$ conditions [15]. The criterion of melting in the calculation is also less ambiguous. However, existing procedures for com-

*tsun@ucas.ac.cn puting $a b$ initio free energies are rather involved, hindering their applications.

As highlighted by earlier studies [12-14], precise prediction of melting properties requires highly accurate free energies. An error of $10 \mathrm{meV} /$ atom shifts the predicted $T_{m}$ by $\sim 100 \mathrm{~K}$ [13]. Accordingly, melting properties are often calculated via thermodynamic integration (TI) [16], a formally exact technique relating the system under study to a reference system whose free energy is known. For classical systems, the procedures to perform TI are well established $[16,17]$, whereas for ab initio systems, conducting TI remains a challenge. Part of the difficulty comes from finding suitable references. In the pioneering works by Alfè et al. [13,14], the references comprise particles interacting with classical potentials specifically fitted to mimic the ab initio systems. Using such $a d$ hoc references reduces the amount of $a b$ initio calculations required for TI. However, it also makes the procedure unduly complicated. Fitting classical potentials is a nontrivial task, especially for complex systems [18]. Besides, the free energy of the reference is not known in advance and a separate TI is needed to evaluate it $[13,14]$. To avoid such complications, simpler and more general references are needed. For the solid phase, a natural choice is the harmonic force field $[12,19,20]$, which can be readily determined via density functional perturbation theory (DFPT) [21] or finite differences [22]. For the liquid phase, a preferable reference is less apparent. Attempts have been made to use a LennardJones fluid [12] or an ideal gas [23] as references. The Lennard-Jones fluid is not a universal choice as in some circumstances the liquid system freezes in the transition [24]. The ideal gas is more general and widely used in classical TI 
of liquids [25-28]. However, it is not suitable for $a b$ initio simulations $[13,14]$. To reach the state of ideal gas, one needs to either expand the system to very large volumes (reversible expansion) [25,26], or gradually switch off the interaction (adiabatic switching) [27,28]. As ab initio systems contain long-range attractive interactions, they cannot be expanded reversibly without discontinuities (phase transitions) [25,26], except for very high $T$ where the system is supercritical. Similarly, when the interaction is critically reduced, atoms can move very close to each other and electronic minimizations break down $[13,14]$. This inhibits evaluating the integrand of TI for all regions and leaves the result indeterminate.

As well as needing a suitable reference for the liquid phase, the size dependence of free energies also requires careful consideration [17]. The liquid phase lacks long-range ordering and the mild size dependence of its free energy is mostly related to the permutation of identical atoms [29]. The free energy of the solid phase converges more slowly with system size because molecular dynamics (MD) simulations only sample phonons whose wavelengths are commensurate to the simulation cell. In general, long wavelength (low frequency) phonons have larger entropies compared to short wavelength (high frequency) phonons. Accordingly, one needs to use very large cells comprising thousands of atoms to get converged free energies [30,31]. Such large cells are impractical for $a b$ initio simulations, where typical numbers of atoms are 100. To overcome this size limitation, Polson et al. [32] introduced a correction to the free energy associated with the fixed center-of-mass (CM). Accounting for this correction significantly improves the agreement between melting properties predicted by MD and those of experiments [17,33]. Alternatively, one may separate the free energy of the solid phase into harmonic and anharmonic parts [12,19,20]. The dominant harmonic part is strongly size dependent, thus it is computed on a dense $\mathbf{q}$-mesh with Fourier interpolated phonon frequencies. The subsidiary anharmonic part is less sensitive to cell size. Accordingly, it is evaluated with a relatively small cell using TI. More recently, a new approach based on the concept of phonon quasiparticles has been proposed $[30,34]$. This approach is computationally less intensive than TI, yet achieves similar accuracy. Moreover, it is able to overcome the finite-size effect and yield free energies in the thermodynamic limit.

Here we present a general scheme to determine melting properties from $a b$ initio free energies. In this scheme, vibrational entropies of the solid phase are determined from its phonon quasiparticle spectra. Free energies of the liquid phase are evaluated via TI using the Weeks-Chandler-Andersen (WCA) gas as reference. The WCA was initially constructed [35] as a reference system for perturbative free energy calculations of liquids. It shares many characteristics with the classic hard-sphere potential, yet, without discontinuities of the latter, is more suitable for MD simulations. The WCA has been widely employed to study the physical properties of liquids. Here we find it is also a good general reference for $a b$ initio TI. To demonstrate the effectiveness of this scheme, we apply it to the melting properties of hexagonal close-packed (hcp) iron at the Earth's ICB. The effect of system size on melting properties is thoroughly analyzed. Moreover, we find that melting properties are sensitive to the pseudo-potential employed in the simulation. The final results are obtained with the pseudo-potential that most resembles the all-electron interaction.

The paper is organized as follows. In Sec. II we summarize the techniques to calculate free energies: phonon quasiparticles, TI, and free energy perturbation. Simulation details are reported in Sec. III. Free energies of individual phases and melting properties are presented in Sec. IV. Conclusions are in Sec. V.

\section{METHODS}

Thermodynamically, melting properties are determined from the Gibbs free energies $(G)$. For simulations conducted in the NVT ensemble, it is more convenient to first compute the Helmholtz free energy $(F)$, then convert $F$ to $G$ via $G=F+P V$. Due to their distinct dynamics, the way to compute $F$ differs for the solid and liquid phases: the former uses phonons in reciprocal space, whereas the latter samples potential energy surfaces in real space. Details of these techniques are described below.

\section{A. Free energy of the solid phase}

Phonons are the fundamental concept in describing thermal properties of crystalline materials. The renowned quasiharmonic approximation (QHA), which treats crystals as assemblies of noninteracting harmonic phonons, is highly effective for many materials when $T$ is less than half of the melting temperature [36]. However, QHA becomes inadequate at high $T$ where anharmonic effects are nonnegligible. A natural extension of QHA accounting for anharmonicity is to replace harmonic phonons with phonon quasiparticles. [37,38] In contrast to their harmonic counterparts, phonon quasiparticles exhibit $T$-dependent frequencies and lifetimes, making them applicable even in strongly anharmonic systems where harmonic phonons are unstable [34,39].

Numerical determination of phonon quasiparticle spectra (PQS) consists of two steps [30,34]. In the first step, one evaluates the power spectra of mode projected velocity autocorrelation functions, i.e. projected vibrational density of states (VDoS), as

$$
\left\langle v_{\mathbf{q} j}^{\star} v_{\mathbf{q} j}\right\rangle_{v}=\int_{0}^{\infty}\left\langle v_{\mathbf{q} j}^{\star}(0) v_{\mathbf{q} j}(t)\right\rangle e^{i 2 \pi v t} d t,
$$

where $v_{\mathbf{q} j}(t)$ is the mode projected velocity at time $t$. Fitting these power spectra yields the frequencies of phonon quasiparticles whose wavelength are commensurate to the simulation cell. Next, an effective harmonic force constant matrix is constructed such that the complete PQS can be determined via Fourier interpolation. Once the PQS is known, the ionic entropy per atom $S_{\text {ion }}$ can be determined as

$$
S_{\text {ion }}=\frac{k_{B}}{N} \sum_{\mathbf{q} j}\left[\left(n_{\mathbf{q} j}+1\right) \ln \left(n_{\mathbf{q} j}+1\right)-n_{\mathbf{q} j} \ln n_{\mathbf{q} j}\right],
$$

where $n_{\mathbf{q} j} \equiv 1 /\left[\exp \left(h v_{\mathbf{q} j} / k_{B} T\right)-1\right]$ is the number of phonons with wave-vector $\mathbf{q}$ and branch index $j . h$ and $k_{B}$ are the Planck and Boltzmann constant, respectively. $v_{\mathbf{q} j}$ is the frequency of phonon quasiparticle at $T . N$ is the total number of atoms, determined as the number of $\mathbf{q}$ vectors times 
the number of atoms in the unit-cell. To ensure convergence, Eq. (2) needs to be evaluated on a dense $\mathbf{q}$-mesh such that $S_{\text {ion }}$ remains constant upon further increases of $N$. The Helmholtz free energy per atom can then be determined as

$$
F_{s}^{\mathrm{PQS}}=U_{k}+F_{\mathrm{el}}-T S_{\text {ion }},
$$

where $U_{k}$ is the kinetic energy per atom. For a finite $N$ atom system with fixed $\mathrm{CM}, U_{k}=\frac{3}{2} \frac{N-1}{N} k_{B} T$, whereas in the $N \rightarrow \infty$ thermodynamic limit, $U_{k}=\frac{3}{2} k_{B} T$. This latter formula is what we use in determining the free energy of an infinite crystal. $F_{\mathrm{el}}$ is the time-averaged electronic free energy per atom [40]. It contains both the potential energy and the entropic contribution from electrons. Note it is incorrect to apply the formula of harmonic free energy,

$$
F_{h}=\frac{k_{B} T}{N} \sum_{\mathbf{q} j} \ln \left[2 \sinh \frac{h v_{\mathbf{q} j}}{2 k_{B} T}\right],
$$

with quasiparticle frequencies to determine the total vibrational free energy due to double counting in the anharmonic energy [41,42]. By contrast, the entropy formula [Eq. (2)] is valid for both harmonic phonons and phonon quasiparticles $[41,42]$.

To crosscheck the results from phonon quasiparticles, we further calculate free energies using TI. The reference of TI is set to be the harmonic force field $[12,19,20]$. The transition path is sampled by a series of MD simulations with interaction

$$
\Phi_{\lambda}=\Phi_{h}+\lambda\left(\Phi-\Phi_{h}\right) .
$$

Here $\Phi$ is the electronic free energy from $a b$ initio calculations, $\Phi_{h}$ is the potential energy from the harmonic force field, $\lambda$ is the coupling parameter ranging from 0 to 1 . The total free energy per atom is then evaluated as

$$
F_{s}^{\mathrm{TI}}=F_{\mathrm{el}}^{0}+F_{h}+F_{a}=F_{\mathrm{el}}^{0}+F_{h}+\frac{1}{N} \int_{0}^{1}\left\langle\Phi-\Phi_{h}\right\rangle_{\lambda} d \lambda,
$$

where $F_{\mathrm{el}}^{0}$ is the electronic free energy per atom of the static lattice, $F_{h}$ is the harmonic free energy determined by Eq. (4), $F_{a} \equiv \frac{1}{N} \int_{0}^{1}\left\langle\Phi-\Phi_{h}\right\rangle d \lambda$ denotes the anharmonic free energy per atom, \langle\rangle$_{\lambda}$ represents the ensemble average with $\Phi_{\lambda}$ being the potential energy of the ensemble.

Equation (6) is formally exact only for the $N$-atom simulation cell. To get the free energy in the $N \rightarrow \infty$ thermodynamic limit, some form of extrapolation or correction is necessary. One effective approach is to consider the size dependence of $F_{h}$ and $F_{a}$ separately. The harmonic free energy per atom in the $N \rightarrow \infty$ limit, $F_{h}(\infty)$, is easily determined on a dense q-mesh with Fourier interpolated phonon frequencies. The size dependence of $F_{a}$ is weak [20], thus it can be approximated as size-independent, i.e., $F_{a}(\infty)=F_{a}(N)$. As such, the free energy per atom of an infinite crystal equals $F_{\mathrm{el}}^{0}+F_{h}(\infty)+F_{a}$. The difference,

$$
\Delta F_{h}(N)=F_{h}(N)-F_{h}(\infty),
$$

quantifies the size dependence of the free energy of the solid phase.

Besides adopting Fourier-intepolated phonon frequencies, the size effect can also be partly compensated by considering the free energy correction from the fixed CM [32], defined as

$$
\Delta F_{\mathrm{cm}}=\frac{k_{B} T}{N} \ln \left(\frac{\Lambda_{\mathrm{cm}}^{3}}{V}\right),
$$

where $\Lambda_{\mathrm{cm}}=h / \sqrt{2 \pi N m k_{B} T}, m$ is the atomic mass, $V$ is the volume per atom. Note Eqs. (7) and (8) are not equivalent and their effects on melting properties are analyzed in Sec. IV.

\section{B. Free energy of the liquid phase}

As mentioned in the Introduction, the main difficulty in performing $a b$ initio TI on liquids is finding a suitable reference. Here we advocate the WCA gas as a general reference for ab initio TI. The WCA is a shifted and truncated variation of Lennard-Jones potential, defined as

$$
\phi_{\mathrm{WCA}}(r)=\left\{\begin{array}{ll}
4 \epsilon\left[\left(\frac{\sigma}{r}\right)^{12}-\left(\frac{\sigma}{r}\right)^{6}\right]+\epsilon, & r \leqslant 2^{1 / 6} \sigma \\
0, & r>2^{1 / 6} \sigma
\end{array},\right.
$$

where $\epsilon$ and $\sigma$ are energy and length parameters, respectively. The WCA has several nice features: (i) it is short ranged, (ii) it is purely repulsive, (iii) it has no discontinuity in energy and force, (iv) it possesses a simple phase diagram with no liquid-gas transition, and (v) it has an established EOS [43,44], making it an ideal reference for $a b$ initio TI. If we denote the interaction along the transition path as

$$
\Phi_{\lambda}=\Phi_{\mathrm{WCA}}+\lambda \Phi,
$$

where $\Phi$ is the electronic free energy from ab initio calculation, $\Phi_{\mathrm{WCA}}$ is the potential energy from WCA, then when $\lambda \rightarrow 0, \Phi_{\lambda}$ becomes WCA, whose repulsive nature prevents the atoms from getting too close. This is crucial for ab initio simulations as electronic minimization cannot proceed under unphysically close atomic separations. By circumventing direct sampling of such phase spaces, the TI also becomes substantially more efficient. When $\lambda \rightarrow 1, \Phi_{\lambda}=\Phi_{\mathrm{WCA}}+\Phi$, and if the minimal atomic distance in MD is larger than the range of the WCA potential $\left(2^{1 / 6} \sigma\right)$, $\Phi_{\mathrm{WCA}}$ will always be zero and in effect $\Phi_{\lambda}=\Phi$. The free energy of the liquid can then be determined as

$$
\begin{aligned}
F_{l}^{\mathrm{TI}} & =F_{\mathrm{WCA}}+\frac{1}{N} \int_{0}^{1}\langle\Phi\rangle_{\lambda} d \lambda \\
& =F_{\mathrm{WCA}}+\frac{1}{N m} \int_{0}^{1} \frac{\langle\Phi\rangle_{\lambda}}{\lambda^{m-1}} d \lambda^{m} .
\end{aligned}
$$

The transformation from $\lambda$ to $\lambda^{m}(m=0.25)$ [45] is purely mathematical to ensure the integrand may be represented by low-order polynomials. Alternatively, $\Phi_{\lambda}$ can be defined as $\Phi_{\lambda}=(1-\lambda) \Phi_{\mathrm{WCA}}+\lambda \Phi$ and the integrand of TI becomes $\left\langle\Phi-\Phi_{\mathrm{WCA}}\right\rangle_{\lambda}$. The first formalism [Eq. (10)] is slightly simpler and we employ it in the present study.

Before the calculations proceed, the two parameters $\epsilon$ and $\sigma$ need to be set to suitable values. There are two general conditions they should meet: (i) the reduced temperature $T^{\star} \equiv k_{B} T / \epsilon$ should be sufficiently large to ensure the WCA system is in the gas phase (ii) the range of the potential, $2^{1 / 6} \sigma$, should be smaller than the minimal atomic distance in MD simulations, yet not too small to be effective. These conditions are satisfied when we set $\epsilon$ and $\sigma$ such that $T^{\star}=$ 1.5 and $\eta=0.1$, where $\eta$ is the packing fraction defined as $\eta \equiv \pi \sigma^{3} / 6 V$ with $V$ the volume per atom. In principle, any $\epsilon$ 
and $\sigma$ that meet the above two conditions are legitimate. We find it convenient to fix them at the same $T^{\star}$ and $\eta$ as one can use a single $F_{\mathrm{WCA}}$ for all simulations.

The free energy of the WCA gas is expressed as

$$
F_{\mathrm{WCA}}=F_{\mathrm{ig}}+F_{\mathrm{WCA}}^{\prime},
$$

where $F_{\text {ig }}$ and $F_{\text {WCA }}^{\prime}$ are the ideal gas and excess free energy, respectively. At $T^{\star}=1.5, \eta=0.1$, the excess free energy per atom $F_{\mathrm{WCA}}^{\prime}=0.454 k_{B} T$ [44]. The ideal gas part is determined as

$$
\begin{aligned}
F_{\mathrm{ig}} & =-\frac{k_{B} T}{N}\left[\ln \left(\frac{\Omega^{N}}{N ! \Lambda^{3 N}}\right)\right] \\
& \approx k_{B} T\left[\ln \left(\frac{\Lambda^{3}}{V}\right)-1+\frac{\ln (2 \pi N)}{2 N}\right],
\end{aligned}
$$

where $\Lambda=h / \sqrt{2 \pi m k_{B} T}, \Omega \equiv N V$ is the volume of the system. The last term, $\Delta F_{\text {stl }} \equiv k_{B} T \ln (2 \pi N) / 2 N$, originates from the Stirling approximation. $\Delta F_{\text {stl }}$ accounts for most of the size-dependence of $F_{l}$ and needs to be included when evaluating melting properties of finite systems. However, it approaches zero when $N \rightarrow \infty$ and does not affect $F_{l}$ in the thermodynamic limit.

\section{Free energy perturbation}

$A b$ initio free energies calculations are computationally intensive. To improve efficiency, one often performs calculations with a setup that is less demanding. Results from this setup can then be refined to better precision by free energy perturbation (FEP) $[13,14,19,20]$. According to FEP [46], the free energy difference between two systems $A$ and $B$ is determined as

$$
F_{A}-F_{B}=-\frac{k_{B} T}{N} \ln \left[\left\langle\exp \left(-\left(\Phi_{A}-\Phi_{B}\right) / k_{B} T\right)\right\rangle_{B}\right],
$$

where $\Phi_{A}\left(\Phi_{B}\right)$ is the energy of the same configuration in system $A(B),\langle\rangle_{B}$ denotes the ensemble average in system $B$. In practice, $B$ is the coarse but efficient setup, $A$ is the more accurate and more demanding one. $F_{B}$ is determined by the aforementioned techniques. The ensemble average is obtained by taking random snapshots from a MD trajectory in $\mathrm{B}$, then evaluating the energies of these snapshots in $A$. Equation (15) is formally exact. Its main difficulty in practice is that the ensemble average \langle\rangle$_{B}$ converges slowly if $A$ and $B$ are very different. When $A$ and $B$ are similar, i.e., the standard deviation of $\left(\Phi_{A}-\Phi_{B}\right)$ is less than $k_{B} T, 30$ to 50 snapshots are sufficient to converge \langle\rangle$_{B}$ to within $2 \mathrm{meV} /$ atom.

\section{SIMULATION DETAILS}

We performed Born-Oppenheimer MD using the PAW method [47] as implemented in VASP [48] at $330 \mathrm{GPa}$ and temperatures ranging from 5600 to $6800 \mathrm{~K}$. At each $T$, the electron occupancy was set according to the Fermi-Dirac distribution and the corresponding electronic free energy was determined by minimizing the Mermin functional [49]. The electron-electron exchange-correlation interaction was treated by the Perdew-Burke-Ernzerhof generalized gradient approximation (PBE-GGA) [50]. There are three PBE potentials shipped with VASP. Each has valence electrons of $8\left(3 d^{7} 4 s^{1}\right)$, $14\left(3 p^{6} 3 d^{7} 4 s^{1}\right)$, and $16\left(3 s^{2} 3 p^{6} 3 d^{7} 4 s^{1}\right)$, respectively. We find the static EOS predicted by PAW-8 and PAW-16 agree well with the all-electron LAPW result, whereas the EOS from PAW-14 exhibits noticeable deviations at high pressures (see Appendix A for details). We therefore focused on PAW-8 and PAW-16. The more efficient PAW-8 was employed for most of the calculations, the more precise PAW-16 was used in FEP to get the final results. The plane-wave cutoff was set to be 400 (750) eV for PAW-8 (16) and the MD time step was fixed at $1 \mathrm{fs}$.

To quantify the size effect, we conducted two sets of simulations involving 64 and 180 atoms. Simulation cells were set to be cubic for the liquid phase and orthorhombic for the hcp phase. The latter were constructed by expanding the 4-atom $C$-centered unit cell of the hcp structure to $4 \times 2 \times 2$ (64 atom) and $5 \times 3 \times 3$ (180 atom) supercells. A $2 \times 2 \times 2$ Monkhorst-Pack [51] k-point mesh sampling was adopted for simulations with 64 atoms, whereas for those involving 180 atoms, a special $\mathbf{k}$ point $(0.25,0.25,0.25)$ was used for the hcp phase, and $\Gamma$-point was used for the liquid phase. Such samplings were sufficient to converge the pressure to within 0.4 (1.5) $\mathrm{GPa}$ and the total energy within 0.5 (6) $\mathrm{meV} /$ atom for 64 (180) atoms. To reach the hydrostatic state of $330 \mathrm{GPa}$ $\left(\sigma_{x x}=\sigma_{y y}=\sigma_{z z}=330 \mathrm{GPa}, \sigma_{y z}=\sigma_{z x}=\sigma_{z y}=0\right)$, we performed constrained [52] NPT simulations [53]. Each NPT simulation lasted 5 to $10 \mathrm{ps}$ to ensure the convergence of the time-averaged cell parameters. These parameters were then used for $N V T$ simulations, with the 180-atom cell proportional to the 64-atom cell so that their densities were identical. The NVT simulations lasted 10 (5) ps for 64 (180) atoms, which was sufficient to converge the time-averaged electronic free energy $F_{\mathrm{el}}$ to $5 \mathrm{meV} /$ atom. The time averaged thermal stresses were nearly hydrostatic (within $0.6 \%$ ) at $330 \mathrm{GPa}$, both for the 64 -atom and the 180 -atom simulations.

Extracting phonon quasiparticles from MD requires harmonic phonons. To this end, we accompany each $N V T$ simulation of the hcp phase with harmonic phonon calculations. In these calculations, the atoms took their equilibrium positions as in the static hep lattice. The electronic temperatures were set to be identical to those in MD to ensure consistency. The resulting harmonic force constants, combined with the MD trajectory, were fed into the DynaPhoPy code [54] to compute PQS and vibrational entropies $S_{\text {ion }}$. To ensure convergence, the PQS were calculated by Fourier interpolation on a dense q-point mesh equivalent to a supercell containing 64000 atoms. Free energies $F_{s}^{\mathrm{PQS}}$ were then determined from Eq. (3).

As a crosscheck to the phonon quasiparticle approach, free energies of the hcp phase were also evaluated by TI. With the harmonic force field as reference, the VASP code allows one to perform MD with the coupled interaction $\Phi_{\lambda}$ defined in Eq. (5). The coupling parameter $\lambda$ was set according to the roots of the $n$th order Legendre polynomial. Once $\left\langle\Phi-\Phi_{h}\right\rangle_{\lambda}$ was determined from MD, the integral $\int_{0}^{1}\left\langle\Phi-\Phi_{h}\right\rangle_{\lambda} d \lambda$ was computed via the Gaussian-Legendre quadrature. Compared to the Newton-Cotes quadrature where $\lambda$ is equally spaced, the Gaussian-Legendre quadrature requires only half as many intermediate points to reach the same precision [55]. Test calculations showed that a six-point quadrature was sufficient to fully converge the integral. 
To determine the free energy of the liquid phase, we revised the VASP code to let it perform MD with the coupled interaction $\Phi_{\lambda}$ as defined in Eq. (10). This revision is straightforward. The WCA potential is short range and the minimumimage convention is sufficient to ensure all the interacting pairs are accounted for. By contrast, long-range potentials would require more involved techniques such as the Ewald sum or face truncation errors [13]. The two parameters in the WCA potential, $\epsilon$ and $\sigma$, were set at the start of the simulation to ensure $T^{\star}=1.5$ and $\eta=0.1$. For instance, at $T=6400 \mathrm{~K} \epsilon=0.3677 \mathrm{eV}$ and $\sigma=1.105 \AA$. The minimal atomic distance extracted from the radial distribution function is $1.40 \AA, \sim 10 \%$ larger than the range of WCA $\left(2^{1 / 6} \sigma=\right.$

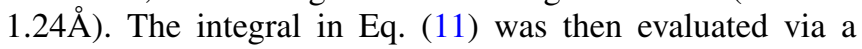
6-point Gaussian-Legendre quadrature, in the same fashion as the solid phase.

The above procedures determine the Helmholtz free energy $F$. To get melting properties, $F$ needs to be transformed to $G$. A caveat in this transformation is that $P$ from MD simulations are not exactly the target pressure $P_{0}(330 \mathrm{GPa})$, but $P \equiv P_{0}+\delta P$. If one uses the definition $G=F+P V$ to conduct the transformation, one gets $G(P)$ instead $G\left(P_{0}\right)$. Even for $\delta P=1 \mathrm{GPa}, G(P)$ and $G\left(P_{0}\right)$ differ by $44 \mathrm{meV}$ at $V=7 \AA^{3}$ /atom, quite significant for determining melting properties. One way to avoid such uncertainties is to perform multiple simulations along the isotherm and get $F\left(V_{0}\right)$ via interpolation, where $V_{0}$ is the volume corresponding to $P_{0}$, then determine $G\left(P_{0}\right)$ as $F\left(V_{0}\right)+P_{0} V_{0}$. However, this would significantly increase the computational cost. We thus took a different approach. Define $\tilde{G}=F(V)+P_{0} V$, where $V \equiv$ $V_{0}+\delta V$, we have

$$
\begin{aligned}
\tilde{G} & =F\left(V_{0}+\delta V\right)+P_{0}\left(V_{0}+\delta V\right) \\
& =F\left(V_{0}\right)+\left.\frac{\partial F}{\partial V}\right|_{V_{0}} \delta V+P_{0} V_{0}+P_{0} \delta V+\mathcal{O}\left(\delta V^{2}\right) \\
& =G\left(P_{0}\right)+\mathcal{O}\left(\delta V^{2}\right) .
\end{aligned}
$$

$\tilde{G}$ is a far better approximation to $G\left(P_{0}\right)$ as the first-order terms in $\delta V$ get canceled. The next order term $\left.\frac{1}{2} \frac{\partial^{2} F}{\partial V^{2}}\right|_{V_{0}} \delta V^{2} \equiv$ $-\frac{\delta P^{2} V_{0}}{2 B_{0}}$, with $B_{0}$ the bulk modulus at $V_{0}$, is tiny $(<$ $0.1 \mathrm{meV} /$ atom) when $\delta P$ is only a few GPa. This perturbative analysis is substantiated by direct calculations along the $T=6400 \mathrm{~K}$ isotherm, where the difference between $\tilde{G}$ and $G\left(P_{0}\right)$ was found to be less than $1 \mathrm{meV} /$ atom. The same trick is applied in computing the enthalpy $H \equiv U+P V$, where the internal energy $U=U_{k}+F_{\text {el }}+T S_{\text {el }}$ with $S_{\text {el }}$ the time-averaged electronic entropy.

Once $G$ is determined from $F$ at one temperature $T_{0}, G$ at other temperatures along the isobar can be determined via

$$
\frac{G}{T}=\frac{G_{0}}{T_{0}}-\int_{T_{0}}^{T} \frac{H}{T^{2}} d T,
$$

where $G_{0}$ is shorthand for $G$ at $T_{0}$. This is more preferable than computing $G$ from $F$ at all temperatures because $H$ is much easier to evaluate than $F$. In practice, we chose $T_{0}=$ $6400 \mathrm{~K}$ and determined $G_{0}$ from $F$, then applied Eq. (17) to obtain $G$ at other temperatures.

\section{RESULTS AND DISCUSSION}

\section{A. Free energy of the solid phase}

As detailed in Secs. II and III, computing free energies of the solid phase involves two key respects: (i) accounting for lattice anharmonicity; (ii) overcoming the finite-size effect and getting free energies in the thermodynamic limit. We first show how these respects are handled in practice using phonon quasiparticles. Then, results from TI are presented as a crosscheck. Finally, we discuss how system size affects the free energies and the preferred routine to reach the thermodynamic limit.

In the framework of phonon quasiparticles, lattice anharmonicity manifests in $T$-dependent phonon frequencies and lifetimes. Both features can be extracted from the mode projected VDoS, as exemplified in Fig. 1(a). Here the low-frequency peak corresponds to the doubly degenerate transverse optical (TO) modes, the high-frequency peak corresponds to the longitudinal optical (LO) mode. These peaks exhibit nice Lorentzian line-shape, indicating well-defined phonon quasiparticles [38]. The TO modes exhibit red shifts, with frequencies decreasing from the harmonic value of 9.75 to $7.09 \mathrm{THz}(-27 \%)$. In contrast, the $\mathrm{LO}$ mode undergoes blue shift, with frequency increasing from the harmonic value of 19.17 to $20.26 \mathrm{THz}$ (+6\%). The large frequency shifts indicate that lattice anharmonicity of hcp iron is quite significant at high $T$.

Mode projected VDoS depict only phonon quasiparticles whose wavelengths are commensurate to the simulation cell. To get the complete PQS, we further construct effective harmonic force constant matrices in real space, from which frequencies of other quasiparticles are determined via Fourier interpolation. This is in line with the standard procedure [56] to determine harmonic phonon dispersion, which also involves constructing harmonic force constant matrices from relatively small supercells and Fourier interpolation for arbitrary phonons. The physics behind such approaches is that atomic interactions decrease rapidly with distance and only those within certain spatial range are significant. Indeed, we find the harmonic phonon dispersions interpolated from the 64-atom and 180-atom force constant matrices are identical, indicating the 64-atom orthorhombic supercell is sufficient to spatially converge the harmonic interaction [57]. As anharmonic interactions are shorter range than their harmonic counterpart [37], the 64-atom supercell should also be good for effective harmonic force constants. Figure 1(b) compares the dispersions of phonon quasiparticles and harmonic phonons interpolated from the 64-atom force constant matrices. We see that most phonon quasiparticles, except those of highest frequencies in the LO branch, have lower frequencies than their harmonic counterparts. This trend is prominent also in the interpolated VDoS as shown in Fig. 1(c). The predominant red-shifts in phonon frequencies increase the overall ionic entropy. At $6400 \mathrm{~K}$, the harmonic entropy is $10.15 k_{B} /$ atom, whereas the entropy of phonon quasiparticles is $10.48 k_{B}$ /atom. Note it is critical to use the converged PQS to compute entropies, as the original VDoS of the 64 atom cell [blue dashed line in Fig. 1(c)] is very different from the interpolated VDoS. The ionic entropy associated with the 64-atom cell (without interpolation) is $10.25 k_{B}$ /atom, 
(a)
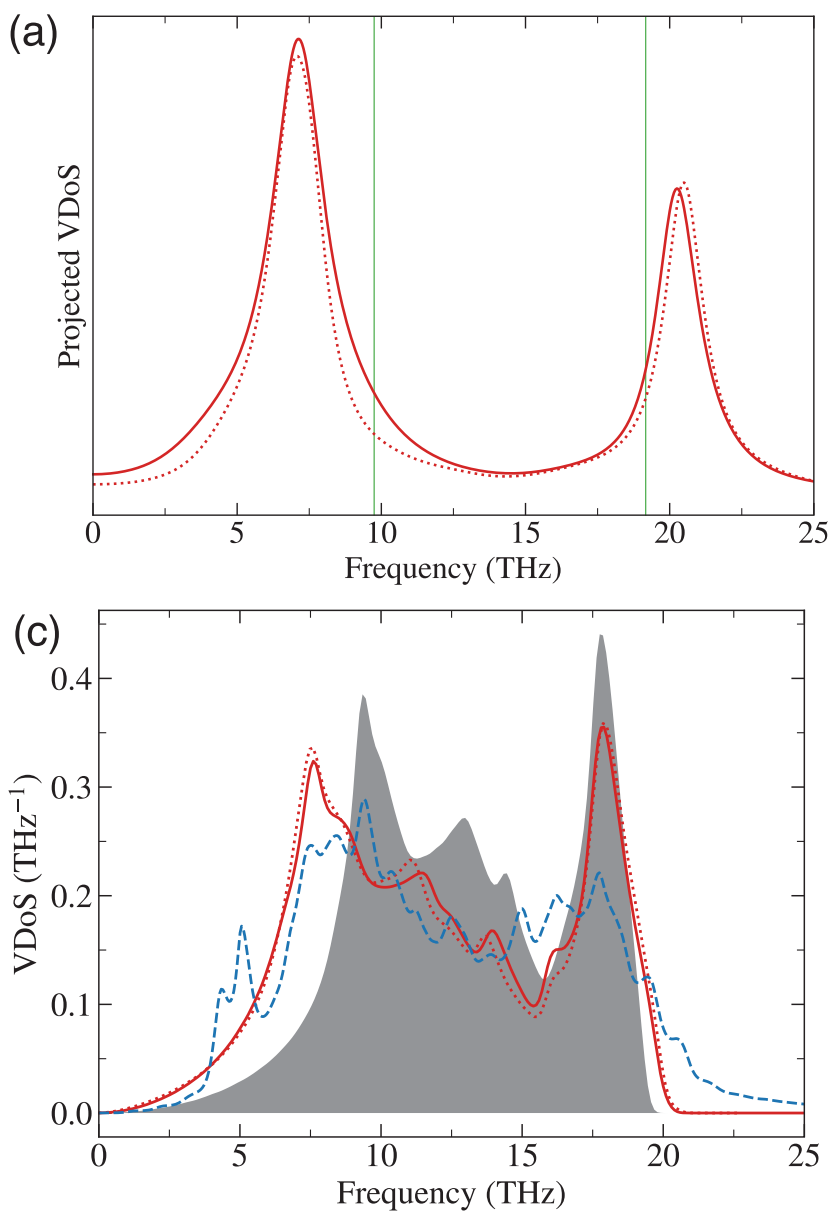

(b)

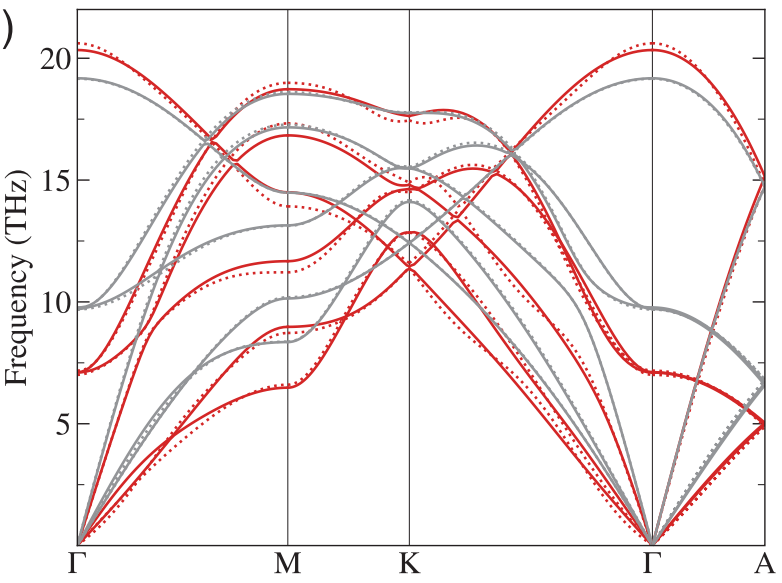

(d)

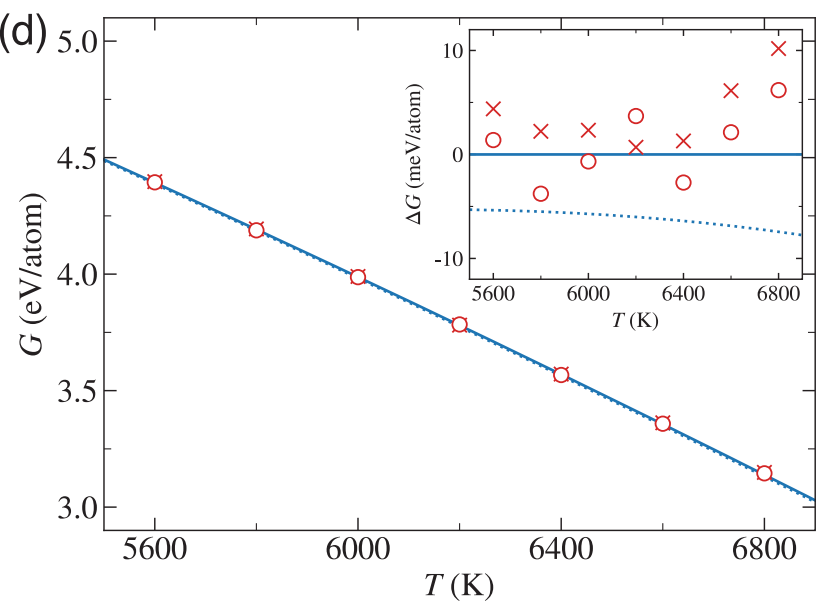

FIG. 1. Phonon quasiparticles of hep iron at $V=6.957 \AA^{3} /$ atom and $T=6400 \mathrm{~K}$. (a) Mode projected VDoS at $\Gamma[\mathbf{q}=(000)]$. Vertical lines correspond to the frequencies of harmonic TO (left) and LO (right) phonons. Results from the 64 (180)-atom cell are denoted by red solid (dotted) lines, respectively. (b) Dispersions of phonon quasiparticles (red) and harmonic phonons (gray). Those from force constant matrices of 64 (180)-atom supercell are denoted by solid (dotted) lines, respectively. (c) Complete VDoS of phonon quasiparticles, where the effective harmonic force constant matrices are from MD involving 64 atoms (red solid line) and 180 atoms (red dotted line). Gray area denotes the VDoS of harmonic phonons, where the harmonic force constant matrix is from the 64-atom supercell. For both phonon quasiparticles and harmonic phonons, Fourier interpolations are performed on a dense q-point mesh equivalent to a supercell containing 64000 atoms. Blue dashed line denotes the VDoS of MD involving 64 atoms without interpolation. (d) The Gibbs free energy per atom $(G)$ in the thermodynamic limit as a function of temperature $(T)$. Red circles (crosses) denote results from the PQS approach using simulation cells with 64 (180) atoms. Blue solid (dotted) lines denote results from TI using 64 (180) atoms. Inset: the differences with respect to $G$ from TI using 64 atoms. Uncertainties in $G$ from phonon quasiparticles are estimated from the variance of five independent simulations at $6400 \mathrm{~K}$ as 6 (4) $\mathrm{meV} / \mathrm{atom}$ for the 64 -atom (180-atom) simulations, respectively. Uncertainty associated with TI is estimated to be $3 \mathrm{meV} /$ atom.

$0.23 k_{B}$ /atom lower than the converged value. As the entropy change at melting is $\sim 1 k_{B}$ /atom, such differences would cause profound changes $(\sim 20 \%, 1000 \mathrm{~K})$ in the predicted $T_{m}$. However, the interpolated VDoS based on effective harmonic force constants of 64 [red solid line Fig. 1(c)] and 180 [red dotted line in Fig. 1(c)] atoms are very similar, with the associated entropies differing by merely $0.015 k_{B}$ /atom. This shows that the 64-atom supercell is indeed sufficient to spatially converge the effective harmonic force constants.

Once the ionic entropies are known, the Gibbs free energies $(G)$ at $P_{0}=330 \mathrm{GPa}$ are readily determined via Eqs. (3) and (16). To check the size dependence, calculations were conducted with 64 and 180 atoms, respectively. The results are summarized in Fig. 1(d). At all temperatures, the differences between the 64- and 180-atom simulations are tiny (within
$8 \mathrm{meV} /$ atom). With Fourier interpolated PQS in the thermodynamic limit, free energies from the phonon quasiparticle approach are insensitive to the size of the simulation cells, insofar as they are sufficiently large to spatially converge the harmonic force constant matrix.

To verify these predictions from phonon quasiparticles, we perform TI using the harmonic force field as reference. In this approach, the free energy is separated into the harmonic $\left(F_{\mathrm{h}}\right)$ and anharmonic $\left(F_{\mathrm{a}}\right)$ parts. $F_{\mathrm{h}}$ in the thermodynamic limit is readily calculated from the interpolated harmonic phonon spectrum using Eq. (4); $F_{a}$ is evaluated via TI using Eqs. (5) and (6). Previous studies [20] have found that $F_{a}$ does not depend strongly on system size. Our simulations confirm this. As shown in Fig. 2(a), the integrands from the 64-atom cell and 180-atom cell are very similar. Once the free energy at 


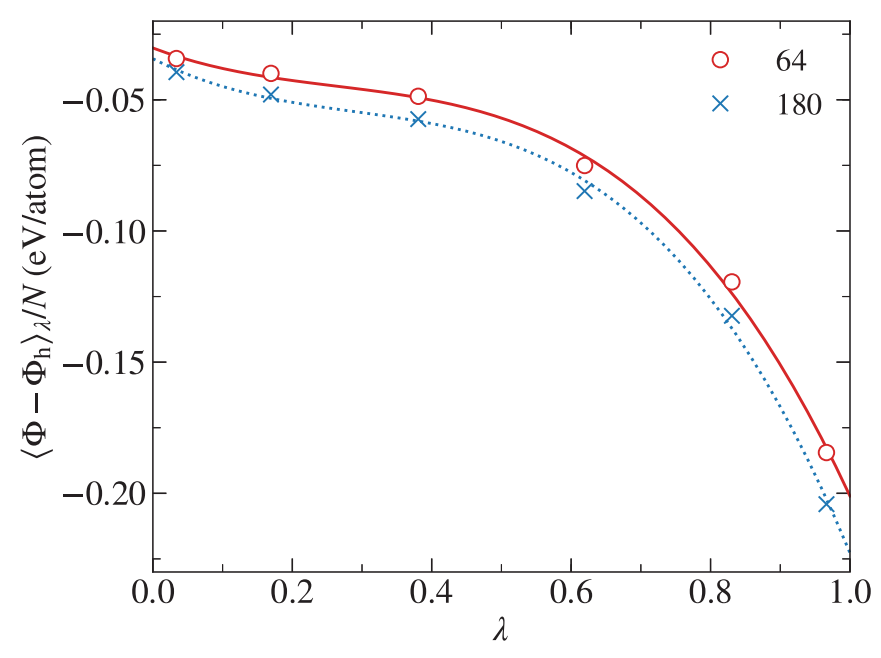

(a)

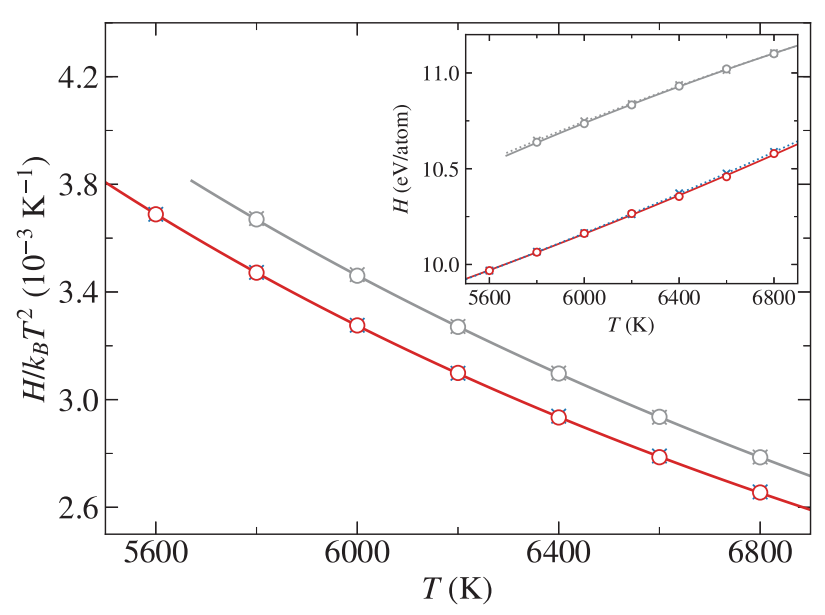

(b)

FIG. 2. (a) The integrand of TI along the transition path at $V=$ $6.957 \AA^{3}$ /atom and $T=6400 \mathrm{~K}$. (b)The $H / k_{B} T^{2}$ as a function of $T$. It is used in Eq. (17) to determine $G$ along the isobar. Inset: $H$ as a function of $T$. Colored (gray) symbols denote data associated with the solid (liquid) phase. Circles and solid lines (crosses and dotted lines) denote results from simulations involving 64 (180) atoms.

$6400 \mathrm{~K}$ is known, $G$ along the isobar is obtained by integrating over $H / T^{2}$, as shown in Fig. 2(b). The results [blue solid and dashed lines in Fig. 1(d)] again exhibit little size dependence (within $10 \mathrm{meV} /$ atom) and are in very good agreement with those from phonon quasiparticles.

Besides Fourier interpolation, a separate strategy to overcome the finite size effect is to take into account the free energy associated with the center of mass [32]. This strategy is mostly used in simulations where the reference system of TI is an Einstein crystal with a single predefined frequency $[17,23,33]$. The Einstein crystal is a simpler reference compared to the harmonic force field; however, it does not allow one to perform Fourier interpolation. Figure 3 compares the free energy associated with $\Delta F_{\mathrm{CM}}$ and the change in the harmonic free energy $\Delta F_{\mathrm{h}}$ for various cell sizes. We see that for systems with 64 atoms, $\Delta F_{\mathrm{CM}}$ and $\Delta F_{h}$ are -0.162

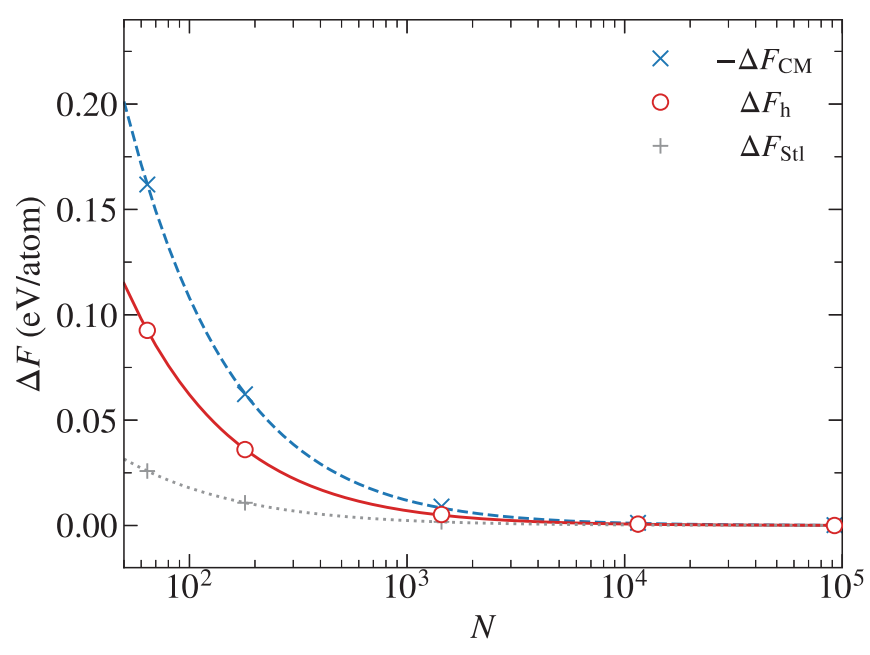

FIG. 3. Size dependence of free energies at $T=6400 \mathrm{~K}$.

to $0.093 \mathrm{eV} /$ atom, respectively. Free energy corrections of such magnitudes can significantly affect melting properties (see Appendix B for more details). The inclusion of $\Delta F_{\mathrm{CM}}$ compensates $\Delta F_{\mathrm{h}}$, however they are not equivalent. Only for large systems $(N>1000)$ are their effects indistinguishable. For $a b$ initio simulations with limited number of atoms $(\sim$ $100)$, Fourier interpolation on harmonic phonons or phonon quasiparticles is the preferred routine to reach the thermodynamic limit.

\section{B. Free energy of the liquid phase}

In contrast to the solid phase, the liquid phase contains substantial atomic diffusion which inhibits a phonon description. The free energy of the liquid phase is therefore calculated differently with key issues as (i) the choice of the reference system; (ii) the size dependence of the free energy. We first describe the main features of the simulation when WCA serves as reference, demonstrating that this choice is indeed appropriate. We then analyze how various free energy components depend on system size.

With WCA as reference, the transition potential $\Phi_{\lambda}=$ $\Phi_{\mathrm{WCA}}+\lambda \Phi$, where $\Phi$ and $\Phi_{\mathrm{WCA}}$ are the ab initio and WCA potential energy, respectively. Figure 4(a) shows the time evolution of $\Phi$ and $\Phi_{\mathrm{WCA}}$ at coupling parameters $\lambda_{i} \equiv(0.5+$ $\left.0.5 \xi_{i}\right)^{1 / m}$, where $m=0.25, \xi_{i}$ is the $i$ th root (in descending order) of the 6-order Legendre polynomial. Specifically, $\lambda_{2}=0.4759677$ and $\lambda_{6}=1.299809 \times 10^{-6}$. For all $\lambda_{i}, \Phi$ and $\Phi_{\mathrm{WCA}}$ do not exhibit discontinuities, indicating that there is no phase transition in the simulation. At $\lambda_{2}$, forces from the $a b$ initio part of the potential $(\lambda \Phi)$ are less than half of the values given by $\Phi$. Nevertheless, they are strong enough to keep the atoms outside the range of WCA. Accordingly, $\Phi_{\mathrm{WCA}}$ remains zero during the simulation. At $\lambda_{6}$, forces from $\lambda \Phi$ are minuscule and the repulsive WCA potential comes into effect, preventing the atoms from getting too close. This allows electronic minimization to proceed and $\Phi$ to be accurately determined. With $\lambda_{6} \sim 10^{-6}, \lambda \Phi$ is tiny and $\Phi_{\lambda}$ is dominated by $\Phi_{\mathrm{WCA}}$. The time average $\left\langle\Phi_{\lambda}\right\rangle / N$ equals $33.9 \pm 0.3 \mathrm{meV} /$ atom, in excellent agreement with the reported potential energy $\left(0.092 \epsilon\right.$ at $T^{\star}=1.5$ and $\eta=0.1$, or 


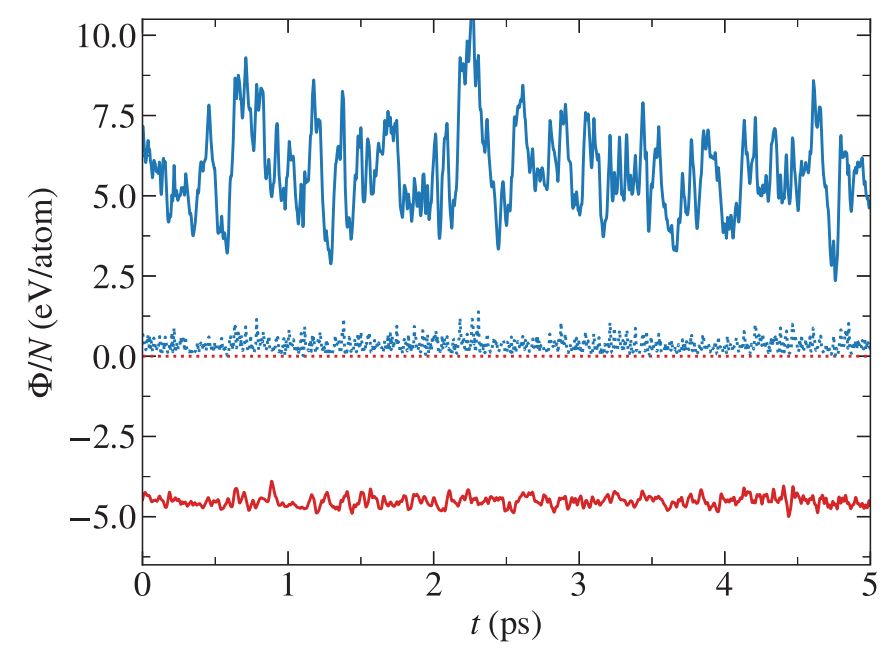

(a)

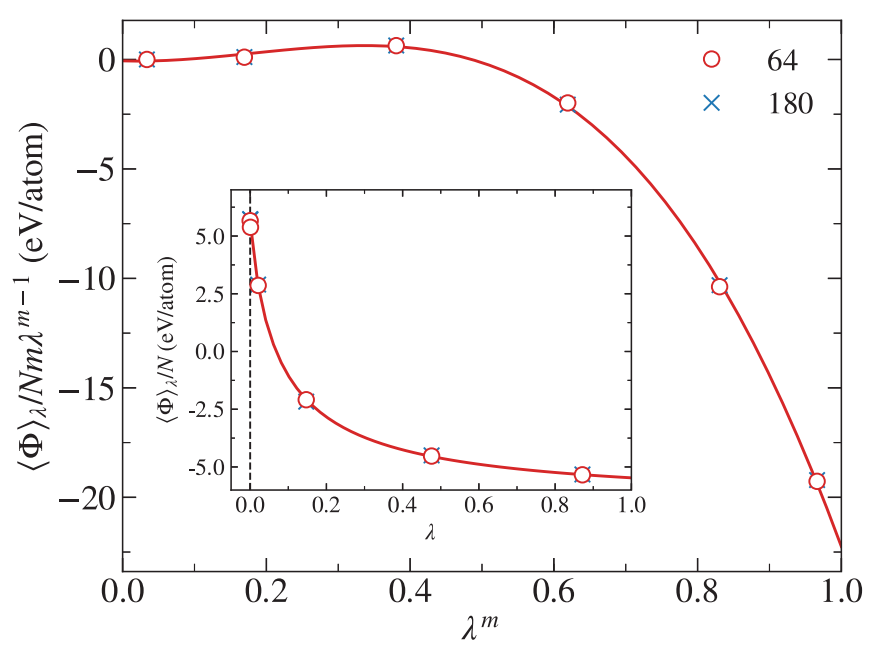

(b)

FIG. 4. TI of the liquid phase at $V=7.063 \AA^{3} /$ atom and $T=$ $6400 \mathrm{~K}$. (a) Time evolution of $\Phi$ (solid line) and $\Phi_{\mathrm{WCA}}$ (dotted line) at $\lambda_{2}$ (red) and $\lambda_{6}$ (blue). As $\Phi_{\mathrm{WCA}}$ is close to zero, for clarity it is multiplied by a factor of 10 . (b) The integrand of TI after integral transformation. Inset: the original integrand as a function of $\lambda$.

$33.8 \mathrm{meV} /$ atom) of WCA gas [44]. In summary, for large $\lambda$ the atoms are outside the range of WCA and the system is fully $a b$ initio; for $\lambda$ close to zero, the system is essentially WCA gas. The transition from ab initio liquid to WCA gas is continuous with no sign of phase transition, which can be attributed to the short range and purely repulsive nature of the WCA potential.

Figure 4(b) shows the time-averaged $a b$ initio potential energy at different $\lambda$. The overall shape of the integrand is similar to those of classical simulations using ideal gas as reference $[27,28]$. After all, a dilute WCA gas is thermodynamically not very different from ideal gas. Moreover, the data from the 64- and 180-atom simulations overlap, indicating that within uncertainties the excess free energy of the liquid phase is independent of system size.
TABLE I. Free energy differences with respect to PAW-8 at $6400 \mathrm{~K}$ (in $\mathrm{meV} /$ atom). $\delta F_{s}\left(\delta F_{l}\right)$ correponds to the free energy difference of the solid (liquid) phase. $\delta F_{l s}$ equals $\delta F_{l}-\delta F_{s}$. Note PAW and LAPW calculations were conducted with two different codes whose basal energies are quite different. For convenience, all the energies from the LAPW calculations are shifted by a constant such as $\delta F_{s}$ equals 0 .

\begin{tabular}{lccc}
\hline \hline & $\delta F_{s}$ & $\delta F_{l}$ & $\delta F_{l s}$ \\
\hline PAW-14 & $57(1)$ & $136(2)$ & $79(3)$ \\
PAW-16 & $-100(2)$ & $-53(1)$ & $47(3)$ \\
LAPW & 0 & $44(2)$ & $44(4)$ \\
\hline \hline
\end{tabular}

While system size has little effect on the excess free energy, it remains relevant to the total liquid free energy due to the $\Delta F_{\text {stl }}$ term in $F_{\text {ig }}$, as defined in Eq. (13). A comparison of $\Delta F_{\text {stl }}$ with the $N$-dependent terms for the solid phase is shown in Fig. 3. At $N=64, \Delta F_{\text {stl }}$ equals $0.026 \mathrm{eV} /$ atom, appreciable but smaller than $\Delta F_{h}(0.093 \mathrm{eV} /$ atom $)$ and $\Delta F_{\mathrm{CM}}(-0.162 \mathrm{eV} /$ atom $)$. All these terms approach zero in the thermodynamic limit and do not contribute to the free energies of macroscopic materials. However, they are important for finite systems with hundreds of atoms. Further analysis can be found in Appendix B.

Once the free energy at $6400 \mathrm{~K}$ is determined, $G(T)$ along the isobar are determined by integrating over $H / T^{2}$ [Fig. 2(b)], in the same fashion as the solid phase. Note in the temperature range we are considering, $H$ of the liquid phase is 0.5 to $0.6 \mathrm{eV} /$ atom higher than the solid phase. Nearly half of this difference comes from the internal energy: the liquid phase is more disordered and has a higher potential energy. The other half is from the pressure-volume term as the volume of the liquid phase is $\sim 1.6 \%$ larger than the solid phase. The enthalpy difference at $T_{m}$ (latent heat) will be discussed later with other melting properties.

\section{From 8-valence to 16-valence}

The aforementioned results are obtained with the PAW- 8 potential. At high pressures, semicore electron orbitals $(3 \mathrm{~s}$ and $3 p$ ) of neighboring atoms can overlap and contribute to metallic bonding. To quantify this effect, we perform FEP using PAW-14 and PAW-16. The results are listed in Table I. The inclusion of semi-core electrons stabilizes the solid phase with respect to the liquid phase by $47 \mathrm{meV} /$ atom for PAW-16 and $79 \mathrm{meV} /$ atom for PAW-14. Such differences far exceed the uncertainty $(10 \mathrm{meV} /$ atom $)$ in the free energy method itself, and would increase the predicted $T_{m}$ by $7 \%$ and $12 \%$, respectively. In contrast, the thermal EOS predicted by PAW-8 and PAW-16 are very similar, with pressures at $\sim 7 \AA^{3}$ /atom and $6400 \mathrm{~K}$ differing by less than $1 \%$. It seems that compared to EOS, melting properties are more sensitive to the chosen pseudopotentials.

To identify the PAW potential whose results are most similar to all-electron calculations, we performed FEP using the full-potential linearized augmented plane wave (FP-LAPW) method as implemented in the wien $2 \mathrm{k}$ code [58]. In this calculation, the $3 s, 3 p, 3 d$, and $4 s$ electrons were treated as valence, the rest were treated as core electrons. The $R K_{\max }$ 
TABLE II. Melting properties of hcp iron under inner core conditions ( $P=330 \mathrm{GPa})$.

\begin{tabular}{|c|c|c|c|c|c|}
\hline & PAW-16 & PAW-8 & Alfè $e t a l .^{\text {a }}$ & Laio et al. ${ }^{\text {b }}$ & Zhang et al. ${ }^{\mathrm{c}}$ \\
\hline$T_{m}(\mathrm{~K})$ & $6170(200)$ & $5730(200)$ & 6350 & 5400 & 6345 \\
\hline$\Delta S_{m}\left(k_{B} /\right.$ atom $)$ & $1.09(3)$ & $1.14(3)$ & 1.05 & 0.86 & 0.87 \\
\hline$\Delta \rho_{m} / \rho(\%)$ & 1.7 & 1.6 & 1.8 & 1.6 & 1.8 \\
\hline$\frac{d T_{m}}{d P}(\mathrm{~K} / \mathrm{GPa})$ & $8.1(2)$ & $6.9(2)$ & 9.0 & 10.0 & 11.0 \\
\hline
\end{tabular}

${ }^{a}$ Ref. [13].

${ }^{\mathrm{b}}$ Ref. [59].

${ }^{\mathrm{c}}$ Ref. [60].

for basis expansion was set to be 10.0, with muffin-tin radius $R$ equaling 1.5 Bohr. The electronic temperature and k-mesh sampling were the same as those of the PAW calculations. We find that $\delta F_{l s}$ from LAPW is nearly identical to that of PAW16 , indicating that PAW-16 closely resembles the all-electron potential. Accordingly, we perform FEP using PAW-16 for all thermal states. The results are then used to determine melting properties.

\section{Melting properties}

The melting properties of iron under inner core conditions have great significance in Earth science [11]. For instance, $T_{m}$ is critical for inferring the temperature at the center of the Earth [6], the latent heat released from the growth of inner core is a main contributor to the Earth's total heat budget [61] and, together with the Clapeyron slope $d T_{m} / d P$, is a key parameter in generating the Earth's magnetic field [62]. We first present our results, then compare with experiments and previous simulations.

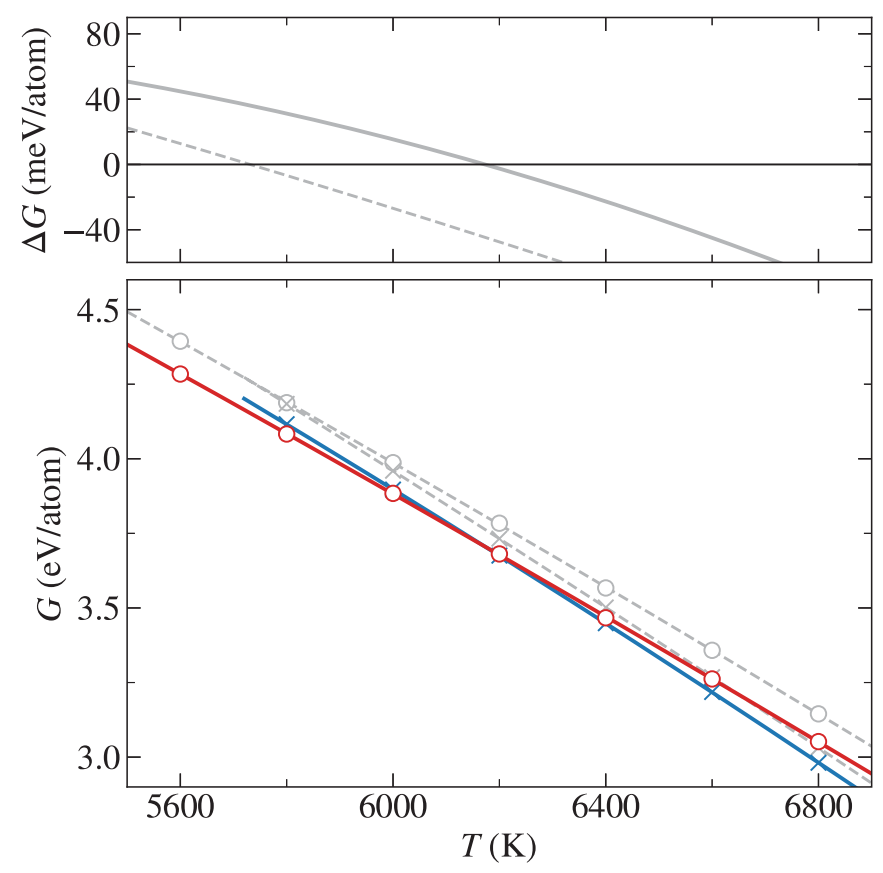

FIG. 5. Gibbs free energies of the hcp (circles) and liquid (crossed) phases from PAW-8 (dashed lines) and PAW-16 (solid lines) potentials.
Figure 5 shows the Gibbs free energies of the liquid $\left(G_{l}\right)$ and solid $\left(G_{s}\right)$ phases at $330 \mathrm{GPa}$. The melting temperature $T_{m}$ is determined from the intercept of $\Delta G=G_{l}-G_{s}$. As shown in the upper panel of Fig. 5, $T_{m}$ predicted by PAW-16 is $6170 \mathrm{~K}$. Since the uncertainties in the free energy calculation is $\sim 10 \mathrm{meV} /$ atom, the uncertainty in the predicted $T_{m}$ is about $\pm 200 \mathrm{~K}$. The change in slopes $\partial G / \partial T$ corresponds to the entropy change $\Delta S_{m}$, from which the latent heat $\Delta H_{m}$ is evaluated as $\Delta H_{m}=T_{m} \Delta S_{m}$. The density change $\Delta \rho_{m} / \rho$ is determined by comparing the thermal EOS of the liquid and solid phases. The Clapeyron slope is then evaluated from $d T_{m} / d P=\Delta V_{m} / \Delta S_{m}$. These results, as well as those from previous studies, are tabulated in Table II.

Experimentally, the $T_{m}$ of iron at the ICB has not been well constrained [11]. Extrapolations from low pressure diamond anvil cell (DAC) measurements yield a relatively low $T_{m}$ $(\sim 4850 \pm 200 \mathrm{~K})$ [6], whereas shock wave measurements give $T_{m} \sim 5800 \pm 600 \mathrm{~K}$ [63]. More recently, DAC measurements based on a new criterion to identify the onset of melting found $T_{m} \sim 6230 \pm 500 \mathrm{~K}$ [7]. Despite the uncertainties associated with PAW potentials, the $T_{m}$ we obtained are within the uncertainties of the shock wave and new DAC experiment and thus support the view that the Earth has a relatively hot core [7].

In comparison to previous simulations, our results are closest to those of Alfè et al. [13]. The small discrepancies are mainly due to the differences in pseudopotentials. Alfè et al. employed a nonstandard PAW consisting of 8 valence electrons and a classical repulsive potential to mimic PAW-14 [64]. The $T_{m}$ it predicts is about $600 \mathrm{~K}$ higher than the value we found for PAW-8 and $200 \mathrm{~K}$ higher than PAW-16. The latent heat values, however, are quite similar as the differences in $T_{m}$ and $\Delta S_{m}$ get canceled. More significant discrepancies are found between $a b$ initio and classical simulations $[59,60]$. This is because $a b$ initio simulations include both electronic and ionic excitations, whereas classical simulations contain only the latter. Accordingly, $\Delta S_{m}$ and $\Delta H_{m}$ from classical simulations are underestimated by about $20 \%$, whereas the Clapeyron slope is overestimated by similar amount. This demonstrates the importance of including electronic excitations in determining melting properties.

\section{CONCLUSIONS}

We have developed a general scheme to accurately determine melting properties of materials from $a b$ initio free energies. In this scheme, free energies of the solid phase 


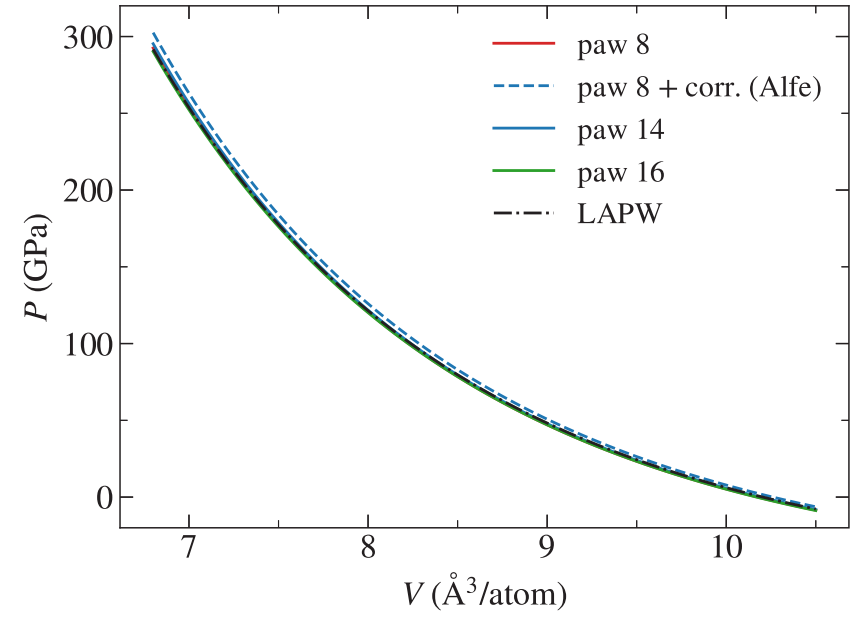

(a)

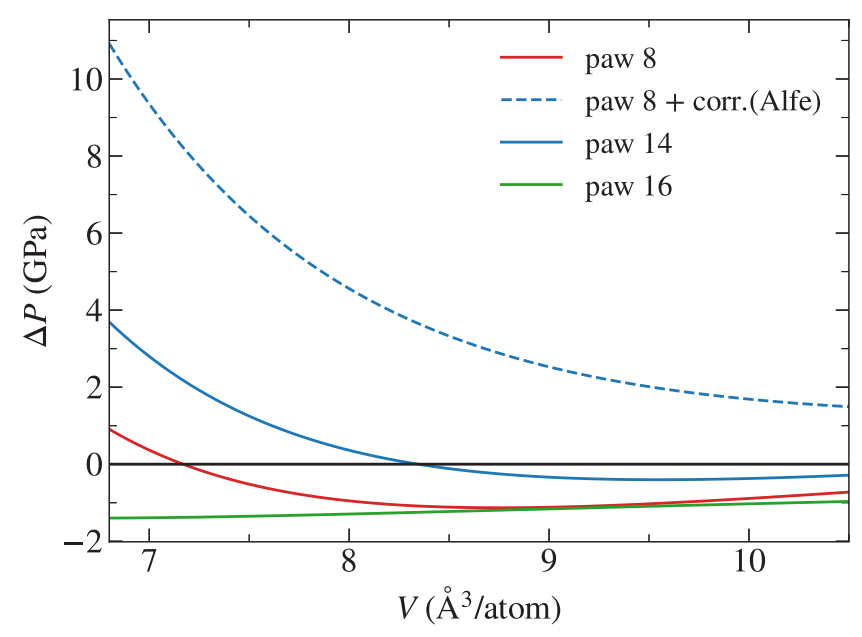

(b)

FIG. 6. Static equation of state of hcp iron determined from different PAW potentials. (a) $P-V$ curves, (b) pressure differences with respect to LAPW calculations.

are determined from phonon quasiparticle spectra, while free energies of the liquid phase are evaluated by TI using the WCA gas as the reference. The scheme is specifically designed for $a b$ initio simulations and allows one to obtain converged results using relatively small simulation cells. Moreover, it avoids the complications associated with fitting system-specific potentials and is well suited for complex materials whose absolute free energies are difficult to evaluate with existing procedures. The effectiveness of this scheme is demonstrated by computing the melting properties of hep iron at the conditions of the Earth's ICB. The predicted $T_{m}$ is in good agreement with shock wave data and recent DAC measurements, confirming that the Earth has a relatively hot core. The refined predictions on latent heat and Clapeyron slope will be useful for geodynamic modeling of the Earth's core.

\section{ACKNOWLEDGMENTS}

We thank Dario Alfè for many helpful discussions. T.S. is supported by the Strategic Priority Research Program (B) of the Chinese Academy of Sciences (Grant No. XDB18000000), MOST Grant No. 2014CB845905, and NSFC Grant No. 41474069. J.P.B., Y.L., and L.V. are supported by NERC Grant No. NE/M015181/1. We also thank the Special Program for Applied Research on Super Computation of the NSFC-Guangdong Joint Fund (the second phase) under Grant No. U1501501. Calculations were performed on ARCHER in UK and Tianhe-2 at the National Supercomputer Center of China (NSCC) in Guangzhou.

\section{APPENDIX A: PAW POTENTIALS}

As a measure of the accuracy of PAW potentials, we calculated the static EOS of hcp iron using a 2-atom primitive cell and a $16 \times 16 \times 16 \mathrm{k}$-mesh sampling. The plane-wave cutoff was set to be $400 \mathrm{eV}$ for PAW-8, and $750 \mathrm{eV}$ for the PAW-14 and 16, respectively. To reach the electronic ground state at $0 \mathrm{~K}$, we employed the Methfessel-Paxton smearing [65] with a width of $0.2 \mathrm{eV}$. As such, the total energy is well-converged
( $<1 \mathrm{meV} /$ atom) and the Pulay stress is negligible $(<0.2 \mathrm{GPa})$. The results are compared with those of Alfè et al. [64] and LAPW calculations by Stixrude et al. [66] as shown in Fig. 6. Alfè et al. employed a nonstandard PAW consisting of 8 valence electrons and a classical repulsive potential. Its EOS exhibits somewhat larger deviation than other PAW potentials. Meanwhile, the EOS of PAW-16 is nearly parallel to that of LAPW with a small difference of $\sim 1 \mathrm{GPa}$, indicating that PAW-16 resembles the all-electron potential quite well.

\section{APPENDIX B: SIZE DEPENDENCE OF MELTING TEMPERATURE}

To better appreciate the effect of finite-size corrections when using thermodynamic integration, here we consider $T_{m}$ of a finite system containing $N$ atoms. Denote the free energy of the solid phase as $F_{s}(N)$, we have $F_{s}(N)=F_{\mathrm{el}}^{0}+$ $F_{h}(N)+F_{a}$, where $F_{\text {el }}^{0}$ is the electronic free energy per atom of the static lattice, $F_{h}(N)$ is the harmonic free energy of the

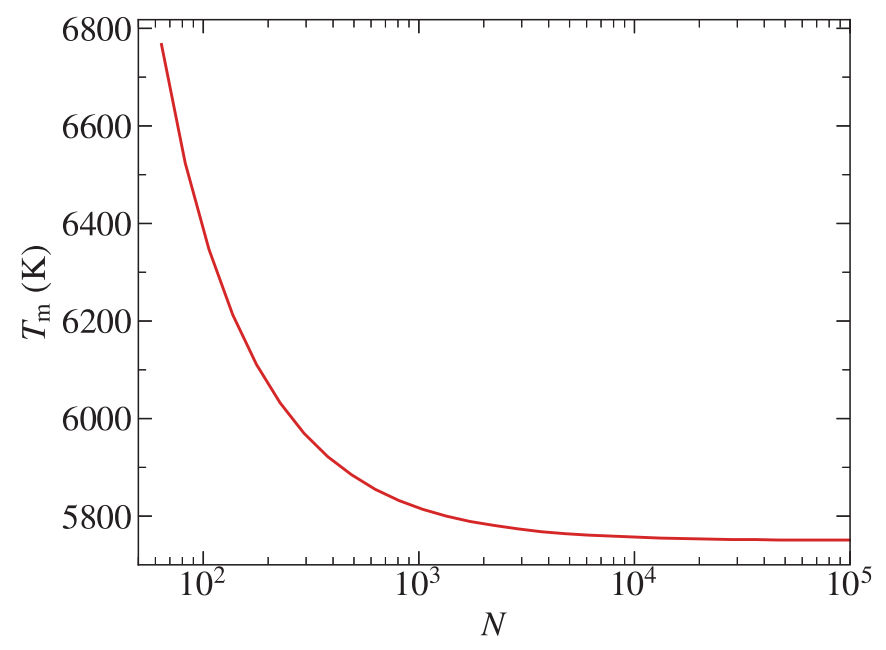

FIG. 7. Size dependence of melting temperature. Free energies are from PAW-8. 
$N$-atom system, $F_{a}$ is the anharmonic free energy. Among these components, only $F_{h}$ exhibits strong $N$-dependence. Recall $\Delta F_{h}(N)=F_{h}(N)-F_{h}(\infty), F_{s}(N)$ can be rewritten as $F_{s}(N)=F_{s}^{\mathrm{TI}}(\infty)+\Delta F_{h}(N)$, where $F_{s}^{\mathrm{TI}}(\infty)$ is the free energy of the solid phase in the thermodynamic limit.

In MD simulations, the CM of the system is fixed, and the above $F_{s}(N)$ corresponds to the free energy of a solid with fixed CM. To remove this constraint, a correction $\Delta F_{\mathrm{CM}}(N)$ as defined by Eq. (8) needs to be included. As such,

$$
F_{s}(N)=F_{s}^{\mathrm{TI}}(\infty)+\Delta F_{h}(N)+\Delta F_{\mathrm{CM}}(N) .
$$

Similarly, the free energy of the liquid phase $F_{l}(N)$ can be expressed as

$$
F_{l}(N)=F_{l}^{\mathrm{TI}}(\infty)+\Delta F_{\mathrm{stl}}(N)
$$

[1] P. Haasen, Physical Metallurgy, 3rd ed. (Cambridge University Press, Cambridge, 1996).

[2] K. L. Murty and I. Charit, An Introduction to Nuclear Materials (Wiley-VCH, Weinheim, 2013).

[3] J. P. O'Connell and J. M. Haile, Thermodynamics: Fundamentals for Applications (Cambridge University Press, Cambridge, 2005).

[4] R. Nomura, K. Hirose, K. Uesugi, Y. Ohishi, A. Tsuchiyama, A. Miyake, and Y. Ueno, Low core-mantle boundary temperature inferred from the solidus of pyrolite, Science 343, 522 (2014).

[5] A. R. Thomson, M. J. Walter, S. C. Kohn, and R. A. Brooker, Slab melting as a barrier to deep carbon subduction, Nature 529, 76 (2016).

[6] R. Boehler, Temperatures in the Earth's core from melting-point measurements of iron at high static pressures, Nature 363, 534 (1993).

[7] S. Anzellini, A. Dewaele, M. Mezouar, P. Loubeyre, and G. Morard, Melting of iron at Earth's inner core boundary based on fast X-ray diffraction, Science 340, 464 (2013).

[8] M. M. Hirshmann, M. S. Ghiorso, L. E. Wasylenki, P. D. Asimow, and E. M. Stolper, Calculation of peridotite partial melting from thermodynamic models of minerals and melts. I. Review of methods and comparison with experiments, J. Petrol. 39, 1091 (1998).

[9] G. Fiquet, A. L. Auzende, J. Siebert, A. Corgne, H. Bureau, H. Ozawa, and G. Garbarino, Melting of peridotite to 140 gigapascals, Science 329, 1516 (2010).

[10] E. S. Kiseeva, V. S. Kamenetsky, G. M. Yaxley, and S. R. Shee, Mantle melting versus mantle metasomatism- "The chicken or the egg" dilemma, Chem. Geol. 455, 120 (2017).

[11] R. A. Fischer, Melting of Fe alloys and the thermal structure of the core, in Deep Earth: Physics and Chemistry of the Lower Mantle and Core, edited by H. Terasaki and Rebecca A. Fischer (American Geophysical Union, 2016), p. 1.

[12] G. A. de Wijs, G. Kresse, and M. J. Gillan, First-order phase transitions by first-principles free-energy calculations: The melting of Al, Phys. Rev. B 57, 8223 (1998).

[13] D. Alfè, G. D. Price, and M. J. Gillan, Iron under Earth's core conditions: Liquid-state thermodynamics and high-pressure melting curve from $a b$ initio calculations, Phys. Rev. B 65, 165118 (2002). where $F_{l}^{\mathrm{TI}}(\infty)$ is the free energy of the liquid phase in the thermodynamic limit. Note $F_{l}(N)$ is based on the free energy of $N$-particle ideal gas whose CM is not fixed. The inclusion of $\Delta F_{\mathrm{CM}}(N)$ in $F_{S}(N)$ is crucial to align the free energies of the two phases to the same footing.

Once $F_{s}(N)$ and $F_{l}(N)$ are known, they are converted into the Gibbs free energies $G_{s}(N)$ and $G_{l}(N)$ whose intersection corresponds to $T_{m}$. The results are shown in Fig. 7. At $N=$ 64, $T_{m}$ is about $6700 \mathrm{~K}, 1000 \mathrm{~K}$ higher than $T_{m}$ in the thermodynamic limit. As $N$ increases, $T_{m}$ drops rapidly and reaches a plateau near $N \sim 1000$. To get fully converged $T_{m}$ directly from $\mathrm{MD}$, one needs to employ simulation cells containing more than 1000 atoms. By contrast, the approach we present allows one to get converged results using much smaller simulation cells.

[14] L. Vočadlo and D. Alfè, A $b$ initio melting curve of the fcc phase of aluminum, Phys. Rev. B 65, 214105 (2002).

[15] D. Alfè, L. Vočadlo, G. D. Price, and M. J. Gillan, Melting curve of materials: Theory versus experiments, J. Phys.: Condens. Matter 16, S973 (2004).

[16] D. Frenkel and B. Smit, Understanding Molecular Simulation (Academic Press, San Diego, 2002).

[17] C. Vega, E. Sanz, J. L. F. Abascal, and E. G. Noya, Determination of phase diagrams via computer simulation: Methodology and applications to water, electrolytes and proteins, J. Phys.: Condens. Matter 20, 153101 (2008).

[18] A. R. Oganov, J. P. Brodholt, and G. D. Price, Comparative study of quasiharmonic lattice dynamics, molecular dynamics and Debye model applied to $\mathrm{MgSiO}_{3}$ perovskite, Phys. Earth Planet. Int. 122, 277 (1999).

[19] B. Grabowski, L. Ismer, T. Hickel, and J. Neugebauer, $A b$ initio up to the melting point: Anharmonicity and vacancies in aluminum, Phys. Rev. B 79, 134106 (2009).

[20] S. G. Moustafa, A. J. Schultz, E. Zurek, and D. A. Kofke, Accurate and precise $a b$ initio anharmonic free-energy calculations for metallic crystals: Application to hcp Fe at high temperature and pressure, Phys. Rev. B 96, 014117 (2017).

[21] S. Baroni, S. de Gironcoli, A. Dal Corso, and P. Giannozzi, Phonons and related crystal properties from density-functional perturbation theory, Rev. Mod. Phys. 73, 515 (2001).

[22] A. Togo and I. Tanaka, First principles phonon calculations in materials science, Scr. Mater. 108, 1 (2015).

[23] T. Taniuchi and T. Tsuchiya, The melting points of $\mathrm{MgO}$ up to $4 \mathrm{TPa}$ predicted based on ab initio thermodynamic integration molecular dynamics, J. Phys.: Condens. Matter 30, 114003 (2018).

[24] B. J. Jesson and P. A. Madden, Ab initio determination of the melting point of aluminum by thermodynamic integration, J. Chem. Phys. 113, 5924 (2000).

[25] J.-P. Hansen and L. Verlet, Phase transitions of the lennardjones system, Phys. Rev. 184, 151 (1969).

[26] J. Mei and J. W. Davenport, Free-energy calculations and the melting point of Al, Phys. Rev. B 46, 21 (1992).

[27] M. Mezei, Direct calculation of the excess free energy of the dense Lennard-Jones fluid, Mol. Simul. 2, 201 (1989). 
[28] T. Sun, J. Xian, H. Zhang, Z. Zhang, and Y. Zhang, Twophase thermodynamic model for computing entropies of liquids reanalyzed, J. Chem. Phys. 147, 194505 (2017).

[29] C. Vega and E. G. Noya, Revisiting the Frenkel-Ladd method to compute the free energy of solids: The Einstein molecule approach, J. Chem. Phys. 127, 154113 (2007).

[30] D.-B. Zhang, T. Sun, and R. M. Wentzcovitch, Phonon Quasiparticles and Anharmonic Free Energy in Complex Systems, Phys. Rev. Lett. 112, 058501 (2014).

[31] P. Korotaev, M. Belov, and A. Yanilkin, Reproducibility of vibrational free energy by different methods, Comput. Mater. Sci. 150, 47 (2018).

[32] J. M. Polson, E. Trizac, S. Pronk, and D. Frenkel, Finite-size corrections to the free energies of crystalline solids, J. Chem. Phys. 112, 5339 (2000).

[33] J. Anwar, D. Frenkel, and M. G. Noro, Calculation of the melting point of $\mathrm{NaCl}$ by molecular simulation, J. Chem. Phys. 118, 728 (2003).

[34] T. Sun, D.-B. Zhang, and R. M. Wentzcovitch, Dynamic stabilization of cubic $\mathrm{CaSiO}_{3}$ perovskite at high temperatures and pressures from $a b$ initio molecular dynamics, Phys. Rev. B 89, 094109 (2014).

[35] J. D. Weeks, D. Chandler, and H. C. Andersen, Role of repulsive forces in determining the equilibrium structure of simple liquids, J. Chem. Phys. 54, 5237 (1971).

[36] R. M. Wentzcovitch, Y. G. Yu, and Z. Wu, Thermodynamic properties and phase relations in mantle minerals investigated by first principles quasiharmonic theory, Rev. Mineral. Geochem. 71, 59 (2010).

[37] R. A. Cowley, Anharmonic crystals, Rep. Prog. Phys. 31, 123 (1968).

[38] T. Sun, X. Shen, and P. B. Allen, Phonon quasiparticles and anharmonic perturbation theory tested by molecular dynamics on a model system, Phys. Rev. B 82, 224304 (2010).

[39] Y. Lu, T. Sun, P. Zhang, P. Zhang, D.-B. Zhang, and R. M. Wentzcovitch, Premelting hcp to bcc Transition in Beryllium, Phys. Rev. Lett. 118, 145702 (2017).

[40] R. M. Wentzcovitch, J. L. Martins, and P. B. Allen, Energy versus free-energy conservation in first-principles molecular dynamics, Phys. Rev. B 45, 11372 (1992).

[41] D. C. Wallace, Thermodynamics of Crystals (Wiley, New York, 1972).

[42] P. B. Allen, Anharmonic phonon quasiparticle theory of zeropoint and thermal shifts in insulators: Heat capacity, bulk modulus, and thermal expansion, Phys. Rev. B 92, 064106 (2015).

[43] D. M. Heyes and H. Okumura, Equation of state and structural properties of the Weeks-Chandler-Andersen fluid, J. Chem. Phys. 124, 164507 (2006).

[44] A. Mirzaeinia, F. Feyzi, and S. M. Hashemianzadeh, Equation of state and Helmholtz free energy for the atomic system of the repulsive Lennard-Jones particles, J. Chem. Phys. 147, 214503 (2017).

[45] M. R. Mruzik, F. F. Abraham, D. E. Schreiber, and G. M. Pound, A Monte Carlo study of ion-water clusters, J. Chem. Phys. 64, 481 (1976).

[46] R. W. Zwanzig, High-temperature equation of state by a perturbation method. I. Nonpolar gases, J. Chem. Phys. 22, 1420 (1954).
[47] P. E. Blochl, Projector augmented-wave method, Phys. Rev. B 50, 17953 (1994).

[48] G. Kresse and D. Joubert, From ultrasoft pseudopotentials to the projector augmented-wave method, Phys. Rev. B 59, 1758 (1999).

[49] N. D. Mermin, Thermal properties of the inhomogeneous electron gas, Phys. Rev. 137, A1441 (1965).

[50] J. P. Perdew, K. Burke, and M. Ernzerhof, Generalized Gradient Approximation Made Simple, Phys. Rev. Lett. 77, 3865 (1996).

[51] H. J. Monkhorst and J. D. Pack, Special points for Brillouinzone integrations, Phys. Rev. B 13, 5188 (1976).

[52] For the liquid phase, the constraint was that the cell remained cubic, i.e., only the length of the cell was allow to change. For the solid phase, the cell remained orthorhombic with the b/a ratio fixed to that of the hcp lattice.

[53] E. Hernández, Metric-tensor flexible-cell algorithm for isothermal-isobaric molecular dynamics simulations, J. Chem. Phys. 115, 10282 (2001).

[54] A. Carreras, A. Togo, and I. Tanaka, DynaPhoPy: A code for extracting phonon quasiparticles from molecular dynamics simulations, Comput. Phys. Commun. 221, 221 (2017).

[55] C. Lanczos, Applied Analysis (Prentice-Hall, Upper Saddle River, NJ, 1956).

[56] X. Gonze, J.-C. Charlier, D. C. Allan, and M. P. Teter, Interatomic force constants from first principles: The case of $\alpha$-quartz, Phys. Rev. B 50, 13035 (1994).

[57] See Supplemental Material at http://link.aps.org/supplemental/ 10.1103/PhysRevB.98.224301 for the spatial convergence of harmonic force constant matrices with respect to supercell size.

[58] P. Blaha, K. Schwarz, G. K. H. Madsen, D. Kvasnicka, J. Luitz, R. Laskowski, F. Tran, and L. D. Marks, WIEN2k, An Augmented Plane Wave + Local Orbitals Program for Calculating Crystal Properties (Karlheinz Schwarz, Techn. Universität Wien, Austria, 2018).

[59] A. Laio, S. Bernard, G. L. Chiarotti, S. Scandolo, and E. Tosatti, Physics of iron at Earth's core conditions, Science 287, 1027 (2000).

[60] W.-J. Zhang, Z.-Y. Liu, Z.-L. Liu, and L.-C. Cai, Melting curves and entropy of melting of iron under Earth's core conditions, Phys. Earth Planet. Int. 244, 69 (2015).

[61] T. Lay, J. Hernlund, and B. A. Buffett, Core-mantle boundary heat flow, Nat. Geosci. 1, 25 (2008).

[62] C. Davies, M. Pozzo, D. Gubbins, and D. Alfè, Constraints from material properties on the dynamics and evolution of Earth's core, Nat. Geosci. 8, 678 (2015).

[63] J. M. Brown and R. G. McQueen, Phase transitions, Gruneisen parameter, and elasticity for shocked iron between 77 and 400 GPa, J. Geophys. Res. 91, 7485 (1986).

[64] D. Alfè, G. Kresse, and M. J. Gillan, Structure and dynamics of liquid iron under Earth's core conditions, Phys. Rev. B 61, 132 (2000).

[65] M. Methfessel and A. T. Paxton, High-precision sampling for Brillouin-zone integration in metals, Phys. Rev. B 40, 3616 (1989).

[66] L. Stixrude, R. E. Cohen, and D. J. Singh, Iron at high pressure: Linearized-augmented-plane-wave in the generalized-gradient approximation, Phys. Rev. B 50, 6442 (1994). 